\title{
BMJ Open Detailing sexual outcomes after treatment of localised prostate cancer with focal therapy using various energy sources: protocol for a mixed-methods study
}

\author{
Gaelle Fiard (D) , ${ }^{1,2,3,4}$ Daniel Kelly (D) , ${ }^{5}$ Tet Yap, ${ }^{6}$ Mark Emberton ${ }^{1,2}$
}

To cite: Fiard G, Kelly D, Yap T, et al. Detailing sexual outcomes after treatment of localised prostate cancer with focal therapy using various energy sources: protocol for a mixedmethods study. BMJ Open 2020;10:e045500. doi:10.1136/ bmjopen-2020-045500

\section{- Prepublication history and} additional material for this paper are available online. To view these files, please visit the journal online (http://dx.doi org/10.1136/bmjopen-2020045500).

Received 05 October 2020 Revised 13 November 2020 Accepted 27 November 2020

Check for updates

(C) Author(s) (or their employer(s)) 2020. Re-use permitted under CC BY-NC. No commercial re-use. See rights and permissions. Published by BMJ.

For numbered affiliations see end of article.

Correspondence to Mrs Gaelle Fiard; g.fiard@ucl.ac.uk

\section{ABSTRACT}

Introduction Focal therapy has emerged as a promising treatment option for men with localised prostate cancer. However, most of the evaluation of postoperative function has taken place at a relatively high, non-granular level. Most of the data we use to provide informed consent for our patients is obtained from retrospective series, or derived from prospective studies whose primary outcome was oncological. Finally, most studies have focused on erectile function and overlooked other, presumably important, elements of male sexual function. The present study aims at studying in-depth the sexual consequences of focal therapy with various energy sources.

Methods and analysis This will be a mixed-methods research study based on a retrospective and prospective cohort, recruited in parallel. The retrospective cohort will consist of patients treated with focal irreversible electroporation, and the prospective cohort of patients treated with three focal therapy energies. Participants will be recruited from two UK urology centres, one National Health Service and one private. On consent, patients will fill in self-administered validated questionnaires (International Index of Erectile Function-15 (IIEF-15), Male Sexual Health Questionnaire-Ejaculatory Dysfunction-Short Form (MSHQ-EjD-SF)) and semistructured interviews will be organised to collect patients' expectations and postoperative changes in domains such as erection, ejaculation, orgasm, libido/sexual desire, masculinity/ virility, penile morphology, pain or discomfort, regret, shame, cancer-related stress, overall impact and partner satisfaction. An exploratory thematic analysis will be performed to detail recurring themes that will be grouped into clusters of experiences. We will then be able to find clusters of agreement and disagreement that will be illustrated using exemplar patient quotations.

Ethics and dissemination Ethical approval was obtained (Regional Ethics Committee reference 20/NW/0335), as well as Health Research Authority approval. Results will be published in open-access peer-reviewed journals. Findings will also be translated into patient information resources (leaflets, online information sheets).

Trial registration number ISRCTN11634296; Pre-results.

\section{INTRODUCTION}

Over the last decade, despite progress in surgical techniques (robotics) and
Strengths and limitations of this study

- This will be the first study using qualitative research methods to study in-depth sexual consequences of focal therapy using various energies to treat localised prostate cancer.

- The design of the study, including both patients already treated at various postoperative time points, and patients enrolled prospectively and interviewed before and after treatment will allow for a unique perspective on patients' expectations and the actual effects of focal therapy.

- The concomitant use of self-administered validated questionnaires will help position the study population in regard to previously published quantitative studies.

- Study limitations include the relatively small sample size for quantitative outcomes, absence of baseline evaluation for the retrospective cohort, and the short-term postoperative evaluation for the prospective cohort.

perioperative management (medication), men's ability to recover erectile function after radical prostatectomy has not improved. Over the last decade, the proportion of men who are able to recover erectile function within 24 months after surgery has remained a constant $30 \%{ }^{1}$ During this same period, progress in MRI of prostate cancer has enabled the emergence of both image-guided biopsy and treatments. This novel diagnostic and therapeutic pathway has conferred a step-change in likelihood that men will experience little, if any, deterioration in their genitourinary function as a result of their prostate cancer diagnosis and treatment. ${ }^{2}$

While the stability of men's genitourinary functional status through the process of treatment is something to be celebrated, most of the evaluation of function has taken place at a relatively high, non-granular level. Most of the data we use to provide informed consent for our patients is obtained from 
retrospective-usually single-centre-series, or derived from prospective studies whose primary outcome was oncological. $^{3}$

Moreover, these studies have-to a very large extentfocused exclusively on erectile function. Other, presumably important, elements of male sexual function (orgasmic sensation, ejaculatory function, penile shape, climacturia) have been largely overlooked. There have been some notable exceptions, such as the careful morphometric analysis of penile length following highintensity focused ultrasound and cryotherapy. ${ }^{4}$ However, we have hardly any insight into the detailed consequences of some of the newer energy sources (such as irreversible electroporation) that are being used to treat the prostate in a tissue-selective manner. ${ }^{5}$ Qualitative research methods enable in-depth exploration of a predefined domain and have already been used to collect patients' sexual recovery after whole-gland treatment. $^{6}$

The present mixed-methods study will address this need by evaluating qualitative sexual outcomes after three different focal therapy modalities, to elicit the evolution of the sexual function of preoperatively potent patients in the early postoperative period (3 months) and at various postoperative time points ranging from 3 to 24 months.

\section{METHODS AND ANALYSIS \\ Study design}

This will be a mixed-methods research study based on a retrospective and a prospective cohort. Both cohorts will be recruited in parallel.

The retrospective cohort will consist of patients treated with focal irreversible electroporation, and the prospective cohort of patients treated with three focal therapy energies.

The retrospective arm of the study will allow us to obtain in-depth details of the evolution of the sexual function of patients at various time points up to 2 years after surgery. This cohort of patients will be able to provide perspective and be more likely to be relieved of the stress related to the oncological outcome. Since there will be a significant time gap between the event and the interview, we also decided to include patients prospectively, to ensure the collection of in-depth sexual patient's experiences as they are being experienced.

Validated patient-reported outcomes questionnaires will allow for a further description of the study population and help position and compare the results obtained with other, mostly quantitative, studies. All steps of this study have been designed to be completed remotely.

An overview of the study design is shown in figure 1.

\section{Study outcomes}

Primary outcome measure

Qualitative sexual outcomes gathered using semistructured telephone interviews at 3 months after focal therapy (prospective cohort).
A.

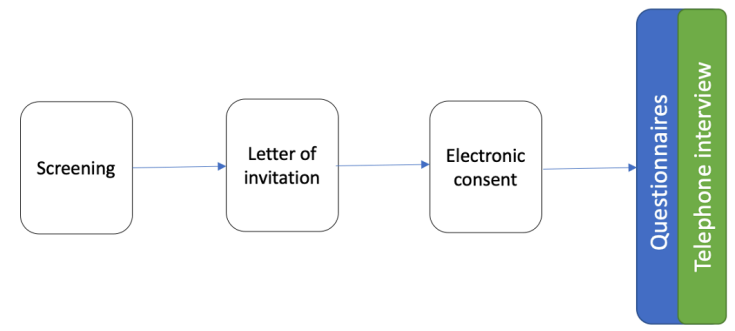

B.
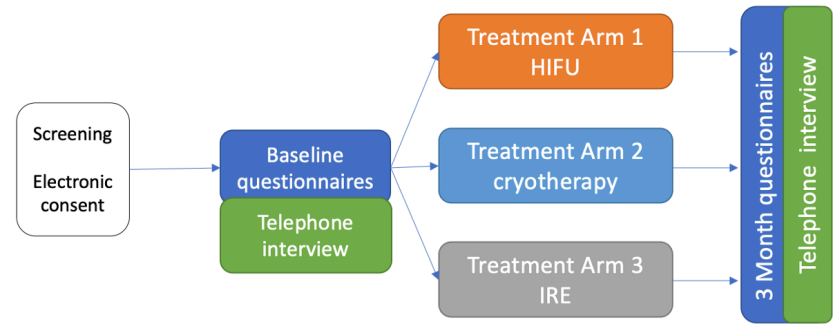

Figure 1 Study overview (A) retrospective cohort; (B) prospective cohort) - no treatment allocation will be made and patients will be treated based on their treating physician's choice, as part of standard care. IRE: irreversible electroporation; HIFU: high intensity focused ultrasound.

\section{Secondary outcome measures}

- Preoperative patients' expectations assessed using semistructured telephone interviews before treatment (prospective cohort).

- Qualitative sexual outcomes gathered using semistructured telephone interviews at various time points ranging from 6 months to 24 months after surgery (retrospective cohort).

- Erectile and ejaculatory function measured by International Index of Erectile Function-15 (IIEF-15) and Male Sexual Health Questionnaire-Ejaculatory Dysfunction-Short Form (MSHQ-EjD-SF) at baseline and 3 months (prospective cohort).

- Erectile and ejaculatory function measured by IIEF-15 and MSHQ-EjD-SF at various postoperative time points (retrospective cohort).

\section{Interview planning}

Semistructured interviews

Interviews will be conducted by the same interviewer (GF) using a predefined interview guide. The interview guide will be defined for all semistructured interviews, with the input of the andrologist and qualitative researcher, part of the study team. Areas to be covered include changes to erections, ejaculation function and pleasure, orgasmic sensation, pain/discomfort, libido/ desire, sense of masculinity/virility, penile morphology (online supplemental material). We will also explore feelings of regret, shame, cancer or sexual related stress, impact on partners and any treatments taken/considered effective. On consent, follow-up phone calls will be performed if new themes arise from the first interviews and result in an amendment of the interview guide. Interviews will be recorded using dedicated hardware and transcribed. 


\section{Validated questionnaires}

Two validated questionnaires, IIEF-15 and MSHQ-EjD-SF exploring sexual and ejaculatory function will be selfadministered prior to the semistructured interviews. The IIEF-15 is a 15-question questionnaire exploring erectile function (questions 1-5 and 15), intercourse satisfaction (questions 6-8), orgasmic function (questions 9 and 10), sexual desire (questions 11 and 12) and overall satisfaction (questions 13 and 14). Scores can be interpreted for each domain or as a whole, with a maximal score of 5 for each question. It has been chosen because it is widely used and validated as a tool to evaluate the sexual consequences of prostate cancer treatment and will allow for a comparison with other studies and treatment modalities. The MSHQ-EjD-SF focuses on the ejaculatory function and contains four questions also each rated on a 5-point scale evaluating the quality and volume of the ejaculate as well as the bother induced by a potential alteration in ejaculation. It has been chosen as the changes expected after focal therapy are likely to be modest and require a specific and precise tool to be accurately captured. Questionnaires will be delivered electronically or through the mail.

\section{Sample size and sampling}

The sample size was determined based on the review of the existing literature on qualitative research in prostate cancer $^{6-8}$ and discussions with qualitative researchers.

\section{Prospective cohort}

The prospective cohort will be recruited using quota convenience sampling to allow for the exploration of sexual outcomes of patients treated using three focal energy modalities: high intensity focused ultrasound (HIFU), cryotherapy and irreversible electroporation. No treatment allocation will be made, and patients will be treated according to their treating physician's choice, as part of standard care. Enrolment will be possible providing preoperative potency (erections sufficient for penetration), electronic consent and sufficient knowledge of English to fill-in the self-administered questionnaires. We aim at recruiting 30 patients in total for the prospective cohort (10 patients for each treatment arm).

\section{Retrospective cohort}

Preoperatively potent patients treated with irreversible electroporation in the last 24 months will be sent an invitation letter. We chose to focus on irreversible electroporation as it is the most recently developed focal therapy energy with the least studied sexual consequences. On electronic consent returned by email, semistructured telephone interviews will be offered to describe qualitative outcomes at agreed time points after surgery. The cohort will be selected purposely so as to represent a variety of postoperative time points (ranging between 3 and 24 months). We aim at recruiting 20 patients for this retrospective cohort.

\section{Study timeline and setting}

The study is set to last for 6 months, with the recruitment of both cohorts being led in parallel. Patients will be recruited from 2 UK urology centres, one National Health Service (University College London Hospital) and one private practice (London Urology Specialists).

\section{Analysis plan}

The interpretation and analysis of data from the qualitative part of the study will be conducted by two members of the research team including a qualitative researcher, both manually and with the help of a designated software (NVivo V.12). An exploratory thematic analysis will be performed to identify recurring themes that then will be grouped into clusters to describe the impact of these treatment experiences. We will then identify areas of agreement and disagreement that will be illustrated using patients' words in quotations. Each transcript will be analysed separately by two researchers from the study team and any disagreement will be addressed by consulting with a third member of the team.

Research will be conducted and reported according to the Consolidated criteria for Reporting Qualitative research statement. ${ }^{9}$

\section{Patient and public involvement}

No patient was involved in the design of this study. A summary of findings written in lay language will be offered to all study participants.

\section{Study status}

Recruitment and data collection are expected to begin in November 2020. Completion of data collection and data analysis is expected to start in August 2021.

\section{DISCUSSION}

Sexual consequences of conventional prostate cancer treatments can impact significantly on men's quality of life. A large survey study including 934 men treated for localised prostate cancer found that significant sexual function bother was reported by $39 \%$ of men and strongly associated with treatment decision regret. ${ }^{10}$ The quality of information we are able to provide patients regarding postoperative outcomes has been shown of major importance to reduce treatment decision regret. ${ }^{11}$ An international multidisciplinary consensus recently concluded that functional outcome assessment was a key component of focal therapy surveillance, but provided little guidance on how this endpoint should be achieved. ${ }^{12}$ The tools commonly used to evaluate patients' post-treatment sexual function (self-administered validated questionnaires such as International Index of Erectile Function -5 (IIEF-5), IIEF15, Expanded Prostate cancer Index Composite (EPIC) scores) have been developed and validated in a population of men treated with whole-gland treatments (surgery, radiotherapy), and provide a gross evaluation, focusing on erection and omitting other aspects such as orgasmic, 
ejaculatory function, sexual desire or other masculinity/ virility issues. Although this might be sufficient to capture major changes expected after conventional, whole-gland treatments, these tools appear to lack depth in capturing more subtle changes after tissue-preserving treatment using focal therapy.

Our study will be, to our knowledge, the first study aiming at analysing in-depth the sexual consequences of focal therapy. The retrospective cohort will help to explore and define the most important components of impact on sexual function directly from men undergoing focal therapy. The prospective cohort approach will identify those domains with a greatest degree of negative impact on sexual function and performance. Importantly, the results will improve the information given to patients before treatment when decision making can be problematic and confusing. ${ }^{13}$ We hope that the depth of detail obtained here will help men to decide on treatment modality with a clear knowledge of the potential consequences of each, particularly for those who consider the preservation of sexual function as a priority.

\section{ETHICS AND DISSEMINATION}

\section{Ethical considerations}

This study deals with a sensitive topic and interviews will be performed outside of standard-of-care clinical follow-up. Informed consent will be sought. Ethical approval was obtained (Regional Ethics Committee reference 20/NW/0335), as well as Health Research Authority approval.

\section{Dissemination}

We plan to communicate the results of this study through abstracts submitted to international conferences, and aim at publishing the results of each cohort in open-access peer-reviewed journals. Findings will also be translated into patient information resources (leaflets, online information sheets).

\section{Author affiliations}

${ }^{1}$ UCL Division of Surgery \& Interventional Science, University College London, London, UK

${ }^{2}$ Department of Urology, University College London Hospital NHS Foundation Trust, London, UK

${ }^{3}$ Department of Urology, Grenoble Alpes University Hospital, Grenoble, France ${ }^{4}$ Université Grenoble Alpes, CNRS, Grenoble INP, TIMC-IMAG, Grenoble, France ${ }^{5}$ School of Healthcare Sciences, Cardiff University, Cardiff, UK

${ }^{6}$ Department of Urology, Guy's and St Thomas' NHS Foundation Trust, London, UK

\section{Twitter Gaelle Fiard @GaelleFiard}

Acknowledgements Gaelle Fiard receives funding from the Fondation de France and the European Urology Scholarship Program. Mark Emberton receives research support from the United Kingdom's National Institute of Health Research (NIHR) UCLH/UCL Biomedical Centre. He was conferred NIHR Senior Investigator status in 2015.

Contributors Manuscript writing: GF. Manuscript editing: DK, TY and ME. Protocol design and writing: GF, DK and TY. Project initiation and supervision: ME.
Funding This work is supported by a grant from Angiodynamics.

Competing interests None declared.

Patient consent for publication Not required.

Provenance and peer review Not commissioned; externally peer reviewed.

Supplemental material This content has been supplied by the author(s). It has not been vetted by BMJ Publishing Group Limited (BMJ) and may not have been peer-reviewed. Any opinions or recommendations discussed are solely those of the author(s) and are not endorsed by BMJ. BMJ disclaims all liability and responsibility arising from any reliance placed on the content. Where the content includes any translated material, BMJ does not warrant the accuracy and reliability of the translations (including but not limited to local regulations, clinical guidelines, terminology, drug names and drug dosages), and is not responsible for any error and/or omissions arising from translation and adaptation or otherwise.

Open access This is an open access article distributed in accordance with the Creative Commons Attribution Non Commercial (CC BY-NC 4.0) license, which permits others to distribute, remix, adapt, build upon this work non-commercially, and license their derivative works on different terms, provided the original work is properly cited, appropriate credit is given, any changes made indicated, and the use is non-commercial. See: http://creativecommons.org/licenses/by-nc/4.0/.

\section{ORCID iDs}

Gaelle Fiard http://orcid.org/0000-0003-3049-5318

Daniel Kelly http://orcid.org/0000-0002-1847-0655

\section{REFERENCES}

1 Capogrosso P, Vertosick EA, Benfante NE, et al. Are we improving erectile function recovery after radical prostatectomy? analysis of patients treated over the last decade. Eur Urol 2019;75:221-8.

2 Valerio M, Cerantola Y, Eggener SE, et al. New and established technology in focal ablation of the prostate: a systematic review. Eur Urol 2017;71:17-34.

3 Yap T, Ahmed HU, Hindley RG, et al. The effects of focal therapy for prostate cancer on sexual function: a combined analysis of three prospective trials. Eur Urol 2016;69:844-51.

4 Li L-Y, Lin Z, Yang M, et al. Comparison of penile size and erectile function after high-intensity focused ultrasound and targeted cryoablation for localized prostate cancer: a prospective pilot study. $J$ Sex Med 2010;7:3135-42.

5 Faure Walker NA, Norris JM, Shah TT, et al. A comparison of time taken to return to baseline erectile function following focal and whole gland ablative therapies for localized prostate cancer: a systematic review. Urol Oncol 2018;36:67-76.

6 Kelly D, Forbat L, Marshall-Lucette S, et al. Co-constructing sexual recovery after prostate cancer: a qualitative study with couples. Transl Androl Urol 2015;4:131-8.

7 Danemalm Jägervall C, Brüggemann J, Johnson E. Gay men's experiences of sexual changes after prostate cancer treatment-a qualitative study in Sweden. Scand J Urol 2019;53:40-4.

8 Wennick A, Jönsson A-K, Bratt O, et al. Everyday life after a radical prostatectomy - A qualitative study of men under 65 years of age. Eur J Oncol Nurs 2017;30:107-12.

9 Tong A, Sainsbury P, Craig J. Consolidated criteria for reporting qualitative research (COREQ): a 32-item checklist for interviews and focus groups. Int J Qual Health Care 2007;19:349-57.

10 Hoffman RM, Lo M, Clark JA, et al. Treatment decision regret among long-term survivors of localized prostate cancer: results from the prostate cancer outcomes study. J Clin Oncol 2017;35:2306-14.

11 Christie DRH, Sharpley CF, Bitsika V. Why do patients regret their prostate cancer treatment? A systematic review of regret after treatment for localized prostate cancer. Psychooncology 2015;24:1002-11.

12 Lebastchi AH, George AK, Polascik TJ, et al. Standardized Nomenclature and surveillance methodologies after focal therapy and partial gland ablation for localized prostate cancer: an international multidisciplinary consensus. Eur Urol 2020;78:371-8.

13 Chhatre S, Wittink MN, Gallo JJ, et al. Sources of information for learning and decision-making in men with localized prostate cancer. Am J Mens Health 2020;14:1557988320945461. 